\title{
Emprego de Simulações Computacionais em Problemas Envolvendo Agricultura: Um Estudo de Mapeamento Sistemático
}

\author{
Dienefer Fialho dos Santos $^{1}$, Fábio Paulo Basso ${ }^{1,2}$, Marcelo Caggiani Luizelli ${ }^{1,2}$, \\ Saimon Martins Cabrera ${ }^{1}$
}

${ }^{1}$ Universidade Federal do Pampa (UNIPAMPA)

${ }^{2}$ Programa de Pós-Graduação em Engenharia de Software (PPGES)

Av. Tiarajú, 810 - Bairro: Ibirapuitã - Alegrete - RS - CEP: 97546-550

\begin{abstract}
The advance promoted by wireless sensor network (WSN) technologies allows monitoring environments in a software-assisted manner. Among the various employment contexts, smart agriculture plays an important role in society, thus a potential for applied studies. However, costs associated with equipment in scenarios involving multiple sensors hampers the exercise of problem-based learning. Studies aimed at computational simulations have the potential to explore the topic without such costs, thus a potential for exploration in problem-based learning. The literature in the area lacks a characterization of the use of computer simulations for problems involving agriculture. This paper presents the results of a systematic mapping study, providing this characterization through 35 analyzed studies.
\end{abstract}

Resumo. O avanço das tecnologias de redes de sensores sem fio (RSSF) permite monitorar ambientes de forma assistida por software. Entre os vários contextos de emprego, a agricultura inteligente desempenha um papel importante na sociedade, caracterizando um potencial para a realização de estudos aplicados. No entanto, os custos associados aos equipamentos em cenários envolvendo diversos sensores dificultam o exercício da aprendizagem baseada em problemas. Estudos voltados para simulações computacionais têm o potencial de explorar o tema sem tais custos, portanto, um potencial de exploração em disciplinas de aprendizagem baseadas em problemas. A literatura da área carece de uma caracterização do uso de simulações computacionais para problemas envolvendo agricultura. Este artigo apresenta os resultados de um estudo de mapeamento sistemático, proporcionando essa caracterização por meio de 35 estudos analisados.

\section{Introdução}

O setor de agricultura é um dos mais avançados no mundo. No entanto, segundo previsões da Organização das Nações Unidas (ONU) até 2050, a população mundial vai chegar a 9.7 bilhões de pessoas, o que cria uma necessidade de aumento de produção de alimentos. O aumento da expectativa de vida e a urbanização global também gera demanda de uma maior variedade de alimentos. Isso exige que a produção agrícola não só aumente como se torne mais eficiente. 
Com tal finalidade, redes de sensores sem fios (RSSF) são usadas em diversas áreas envolvendo monitoramento e controle de ambientes, sendo a agricultura um dos principais setores da economia que é beneficiado por tais tecnologias. RSSFs são, portanto, uma das formas de se buscar uma produção mais eficiente, de melhorar a qualidade dos produtos e reduzir o impacto ambiental das práticas agrícolas (Shinghal et al. 2017).

Neste contexto, a modelagem e implementação de RSSFs enfrenta desafios, não só pela limitação dos dispositivos utilizados, como também a dificuldade de testar as soluções de forma satisfatória antes da implantação. Ou seja, cenários de educação onde os recursos são escassos e os laboratórios não dispõem de equipamentos apropriados, apresentam barreiras para a prática de desenvolvimento de RSSFs. Simulações, por outro lado, permitem a realização de testes próximos do ambiente real sem exigir dispositivos físicos (Khan et al. 2016), tornando viável as práticas educativas em cenários que apresentem tais limitações.

Com o objetivo de fomentar potenciais domínios de RSSFs voltados para a agricultura, e do potencial dos estudos para disciplinas conduzidas na metodologia de Aprendizagem Baseada em Problemas (ABP) (de Souza e Luis Dourado 2015), o presente trabalho apresenta o resultado de um mapeamento sistemático sobre a utilização de simulações computacionais. Na Seção 2 apresenta-se o protocolo do estudo de mapeamento sistemático, seguido do relato de condução e execução na Seção 3, que inclui análise dos resultados e limitações. As conclusões são apresentadas na Seção 4 e, finalmente, os agradecimentos na Seção 5.

\section{Protocolo}

A definição do protocolo de mapeamento foi feita baseada em (Petersen et al. 2008), com a definição das bases de dados para a busca de estudos, as strings de busca, critérios de inclusão e exclusão, e as questões de pesquisa.

Bases e String de Busca Genérica: As bases de dados escolhidas são: IEEE Xplore, Science Direct, Scopus, ACM Digital Library e Springer Link. Utilizou-se a seguinte string base para as buscas com adaptações quando necessário: ("wireless sensor network" OR wsn OR wsan) AND ("internet of things" OR IoT) AND (simulation) AND (agriculture).

Critérios de Seleção: Critério de inclusão: CI01 - os estudos devem tratar de simulações de RSSF para agricultura. Critérios de exclusão: CE01 - trabalhos em idiomas além do Inglês; CE02 - estudos fora do domínio de RSSF; CE03 - artigos indisponíveis ou apenas parcialmente disponíveis para download; CE04 - estudos secundários, e; CE05 - estudos indisponíveis nas bases de dados escolhidas.

\section{Questões de Pesquisa:}

- QP 01 - Para quais ramos da agricultura, IoT e RSSF estão sendo utilizados? O objetivo é caracterizar os estudos pelos seus domínios de aplicação com um mapa que descreve as oportunidades disponíveis para simulações.

- QP 02 - Quais são as soluções técnicas propostas? O objetivo é classificar as tecnologias, métodos e técnicas propostas para os trabalhos.

- QP 03 - Como os estudos implementaram as simulações? Nosso objetivo 
é classificar esses estudos para que se torne possível caracterizar como as simulações estão sendo executadas na área.

- QP 04 - Quais foram os pontos positivos e negativos observados no estudo? O objetivo é caracterizar vantagens e/ou desvantagens que algumas propostas introduziram na área.

- QP 05 - Quais são as lacunas de pesquisa encontradas nos trabalhos? O objetivo é identificar as principais oportunidades de pesquisa na área.

\section{Condução e Execução}

A busca nas bases retornou 699 estudos, foi realizada a remoção de trabalhos repetidos e então foi feita a leitura dos títulos, resumos e palavras-chave utilizando os critérios de inclusão e exclusão, selecionando os artigos para a próxima fase de revisão.

Após a primeira etapa de seleção havia um total de 75 estudos, estes foram lidos por completo. A partir de critérios de qualidade foram selecionados um total de 35 estudos para a realização da extração de dados.

\subsection{Análise de Resultados}

\section{QP 01 - Para quais ramos da agricultura, IoT e RSSF estão sendo utilizados?}

A Tabela 1 apresenta os artigos analisados. Eles englobam uma série de diferentes domínios, variando entre soluções genéricas e outras focadas em um problema bastante específico, sendo caracterizados por domínio na Tabela 2.

Tabela 1. Trabalhos selecionados para análise e extração dos dados

\begin{tabular}{|c|c|c|c|}
\hline ID & Referência & ID & Referência \\
\hline S01 & (Bhanu et al. 2020) & S19 & (Agrawal et al. 2019) \\
\hline S02 & (Wu et al. 2020a) & S20 & (Nicolae et al. 2019) \\
\hline S03 & (Mahalakshmi et al. 2020) & S21 & (Zhang et al. 2019) \\
\hline S04 & (Khelifi 2020) & S22 & (Linsner et al. 2019) \\
\hline S05 & (Iqbal and Butt 2020) & S23 & (Wang et al. 2019) \\
\hline S06 & (Jain 2020) & S24 & (Ibrahim et al. 2018) \\
\hline S07 & (Wu et al. 2020b) & S25 & (Dhall and Agrawal 2018) \\
\hline S08 & (Mukherjee et al. 2020) & S26 & (Jiang et al. 2018) \\
\hline S09 & (Shamshiri et al. 2020) & S27 & (Tanaka et al. 2018) \\
\hline S10 & (Lavanya et al. 2020) & S28 & (Shipu et al. 2018) \\
\hline S11 & (Bayrakdar 2020) & S29 & (Uddin et al. 2018) \\
\hline S12 & (Faid et al. 2020) & S30 & (Uddin et al. 2017) \\
\hline S13 & (Castellanos et al. 2020) & S31 & (Stewart et al. 2017) \\
\hline S14 & (Trotta et al. 2020) & S32 & (Nandhini et al. 2017) \\
\hline S15 & (Banđur et al. 2019) & S33 & (Ojha et al. 2017) \\
\hline S16 & (Yassine et al. 2019) & S34 & (Chen et al. 2015) \\
\hline S17 & (Uddin et al. 2019) & S35 & (Musaazi et al. 2014) \\
\hline S18 & (Khan and Kumar 2019) & \multicolumn{2}{|c|}{} \\
\hline
\end{tabular}

Os domínios com maior número de estudos tratam de Agricultura inteligente e de precisão, que em sua essência já trazem o uso de sensoriamento remoto. Sua natureza genérica permite que diversas pesquisas sejam realizadas dentro deles, o que significa que integram diversos sensores, em ambientes heterogêneos e dinâmicos de culturas e de fontes de dados. São, portanto, ambientes de menor controle, mais difíceis de simulação. 
Tabela 2. Domínios de pesquisa

\begin{tabular}{|c|l|c|}
\hline Domínio & Estudo Mapeado & Total \\
\hline Agricultura de precisão & $\begin{array}{l}\text { S04, S05, S06, S07, S13, S16, S19, S20, S22, S23, S25, S29, S31, S33, } \\
\text { S34, S35 }\end{array}$ & 16 \\
\hline Agricultura inteligente & S01, S03, S08, S10, S11, S12, S14, S15, S17, S30 & 10 \\
\hline Estufa de vegetais & S02, S09, S24, S28 & 4 \\
\hline Irrigação inteligente & $\mathrm{S} 21, \mathrm{~S} 26$ & 2 \\
\hline Cultivo de arroz & $\mathrm{S} 27$ & 1 \\
\hline Campo agrícola & $\mathrm{S} 18$ & 1 \\
\hline Detecção de doenças & $\mathrm{S} 32$ & 1 \\
\hline
\end{tabular}

O domínio de estufa de vegetais inclui diferentes aspectos presentes em agricultura de precisão e inteligente. Porém, por se tratar de um ambiente fechado que oferece maior controle, facilita o uso de sensores.

A área de irrigação inteligente se baseia na utilização de sensores para monitorar as condições da plantação, e assim garantir que a irrigação seja feita de acordo com a necessidade. Ou seja, controlam a água conforme o ambiente monitorado, diferente de outros sistemas automatizados.

O campo agrícola possui mais variáveis de ambiente e oferece oportunidades de avanços com o uso de drones e câmeras. Em um domínio mais específico, o cultivo de arroz atualmente ainda exige muitos recursos, podendo se beneficiar de alternativas mais eficientes de produção.

A detecção de doenças é uma área relevante para todos os domínios de agricultura, encontrar formas de realizar a detecção e controle de doenças é indispensável para obter alimentos de qualidade com maior eficiência.

\section{QP 02 - Quais são as soluções técnicas propostas?}

A análise das soluções técnicas propostas permite caracterizar os problemas encontrados na área de IoT e RSSFs, bem como apontar as tendências para sua resolução.

Em (Khelifi 2020), é feita a proposta de um algoritmo híbrido de rota com o uso de clusters baseados em regiões para a implantação de sensores. Portanto, o problema foco envolve prover uma melhor cobertura para a área onde são utilizados os sensores.

Em (Shipu et al. 2018), propõe-se o uso de algoritmos de roteamento para resolver o seguinte problema: melhorar a comunicação e o consumo de energia em $R S$ SFs. Tal problema se mostra ideal para simular ambientes motivados para domínios de estufas de vegetais, uma vez que se encaixarem melhor no ambiente controlado.

Em (Jiang et al. 2018), apresenta-se a proposta que trata de um problema relevante para avaliar a eficiência em distâncias entre os nós da RSSF. Por exemplo, em domínios de irrigação inteligente, onde os nós da rede necessitam de transmissão de longa distância e de baixo consumo de energia para operarem. Outro problema também derivado na motivação de irrigação é a tecnologia de nuvem de sensores (Zhang et al. 2019).

Um problema comum investigado na literatura é para a detecção e alastramento de doenças. Por exemplo, (Nandhini et al. 2017) explora a simulação num 
sistema web de detecção de doenças para segmentar e classificar doenças em folhas de romã, tomate e berinjela com o uso de wireless multimedia sensor networks (WMSNs).

Outro problema possível de ser tratado em simulação é para identificar vulnerabilidades em RSSFs. Por exemplo, o estudo de (Linsner et al. 2019) apresenta testes de ataques em RSSFs, discutindo sobre questões de segurança na área e como elas afetam os custos de adesão. Outro trabalho neste problema adota um modelo de aplicação de encryption-as-a-service para verificar a comunicação entre nodos via criptografia (Mahalakshmi et al. 2020).

Outro problema para simulação envolve medir parâmetros pela mobilidade das bases de sensoriamento. Por exemplo, a solução proposta por (Khan and Kumar 2019) trata de um sistema de monitoramento de ambiente do campo utilizando uma base móvel para reduzir consumo de energia, delay, e hop count durante transmissão de dados.

Medir a proximidade da modelagem com cenários reais é outro problema possível de estudos exploratórios. Por exemplo, (Wang et al. 2019) e (Wu et al. 2020b) trabalham no desenvolvimento de um simulador para investigar o uso de LoRaWan para RSSFs subterrâneas com o objetivo de permitir uma modelagem mais próxima da implantação.

Por fim, sensoriamento de movimento para controle de invasão também caracterizam potenciais para educação. Por exemplo, em (Iqbal and Butt 2020) os autores propõe um sistema para prevenção de ataques de animais em plantações utilizando os dados coletados por sensores colocados no campo.

\section{QP 03 - Como os estudos implementaram as simulações?}

A seleção de uma ferramenta adequada para realizar a simulação também é do interesse de professores ministrantes de ABP. Nos estudos analisados foram utilizadas 10 ferramentas, alguns trabalhos realizaram simulações em mais de uma delas dependendo da necessidade. A Tabela 3 apresentada a relação de ferramentas por estudos.

Tabela 3. Ferramentas de simulação

\begin{tabular}{|c|c|l|}
\hline Simulação & Total de estudos & Estudos Mapeados \\
\hline Network Simulator & 11 & $\mathrm{~S} 01, \mathrm{~S} 04, \mathrm{~S} 05, \mathrm{~S} 06, \mathrm{~S} 16, \mathrm{~S} 18, \mathrm{~S} 19, \mathrm{~S} 21, \mathrm{~S} 25, \mathrm{~S} 28, \mathrm{~S} 33$ \\
\hline MATLAB & 9 & $\mathrm{~S} 02, \mathrm{~S} 03, \mathrm{~S} 12, \mathrm{~S} 17, \mathrm{~S} 09, \mathrm{~S} 26, \mathrm{~S} 29, \mathrm{~S} 30, \mathrm{~S} 32$ \\
\hline Ferramenta própria & 6 & $\mathrm{~S} 07, \mathrm{~S} 08, \mathrm{~S} 09, \mathrm{~S} 13, \mathrm{~S} 23, \mathrm{~S} 27$ \\
\hline OMNET++ & 5 & $\mathrm{~S} 14, \mathrm{~S} 17, \mathrm{~S} 22, \mathrm{~S} 29, \mathrm{~S} 35$ \\
\hline COOJA & 3 & $\mathrm{~S} 14, \mathrm{~S} 15, \mathrm{~S} 20$ \\
\hline Riverbed Simulator & 2 & $\mathrm{~S} 11, \mathrm{~S} 24$ \\
\hline MSPSim & 1 & $\mathrm{~S} 34$ \\
\hline AGI-STK & 1 & $\mathrm{~S} 17$ \\
\hline OPNET & 1 & $\mathrm{~S} 31$ \\
\hline QualNet & 1 & $\mathrm{~S} 10$ \\
\hline
\end{tabular}

Além das ferramentas de simulação, também foram coletados os parâmetros monitorados pelos estudos. Os sensores principais foram os de ambiente, incluindo parâmetros como umidade, $\mathrm{pH}$, temperatura, luz, salinidade, pesticidas, sereno, dióxido de carbono e pressão do ar. Outros tipos comuns de sensores são os de imagens e drones incluindo sensores de diferentes tipos. 


\section{QP 04 - Quais foram os pontos positivos e negativos observados no estudo?}

No geral, os autores argumentam que o uso de simulações se mostra útil para demonstrar as soluções motivadas, podendo inclusive ser usadas para comparações com outras propostas já existentes na literatura. Simulações são, portanto, muito interessantes para utilização em disciplinas de ABP.

Outro ponto positivo da simulação é que auxiliam na previsão do comportamento do sistema em um ambiente real. Portanto, podem ser utilizadas como alternativas pelos professores em disciplinas curriculares como Resolução de Problemas, que em particular é do interesse de cursos de graduação de Engenharia de Software, Ciência da Computação e Engenharia da Computação.

Apesar de apresentarem casos interessantes para a problematização em ABP, percebe-se uma limitação na literatura da área para servirem como materiais prontos para os estudantes. Por exemplo, a falta de informação disponibilizada sobre as simulações dificulta a compreensão do seu uso na prática do ABP. Por exemplo, o estudo (Tanaka et al. 2018), por ter um carácter mais científico, apresenta como principal contribuição uma ferramenta própria, sem motivar o problema e contextualizar um estudo aplicado. Um contra-exemplo é o estudo (Uddin et al. 2017), que envolveu um cenário composto de três ferramentas de simulação para permitir que diferentes aspectos da proposta fossem simulados.

Outra dificuldade para mapear estudos que possam servir como base para a condução de ABP é identificar os parâmetros dos estudos de simulação. Em alguns estudos como (Agrawal et al. 2019), (Dhall and Agrawal 2018), (Iqbal and Butt 2020), (Khelifi 2020) e (Zhang et al. 2019), os autores oferecem informações sobre os parâmetros definidos, versão do simulador, configurações da máquina e sistema operacional, o que é positivo para a formulação de problemas práticos de desenvolvimento de software. Porém, as informações sobre os parâmetros geralmente estão implícitas, dificultando para professores e cientistas a materialização de problemas.

No geral apesar de existir um bom número de trabalhos utilizando simulações, o que demonstra o potencial da área, percebe-se que não são estudos prontos para utilização em disciplinas de ABP. Em geral, a literatura da área foca na divulgação de suporte ferramental para demonstrar o funcionamento das propostas feitas, não oferecendo uma visão detalhada das técnicas de simulação, que possuem maior valor para ABP.

\section{QP 05 - Quais são as lacunas de pesquisa encontradas nos trabalhos?}

A área apresenta potencial para estudos que sirvam como entrada para professores e cientistas que necessitam educar estudantes com base em problemas. Na execução do mapeamento observou-se uma carência de informações sobre as simulações realizadas nos trabalhos. Algumas exceções são os estudos (Iqbal and Butt 2020), (Zhang et al. 2019), (Khelifi 2020) e (Shipu et al. 2018).

Existe uma carência de estudos que se aprofundem no problema do tema de simulações. Uma vez que o problema costuma ficar em segundo plano, isso dificulta o entendimento e torna difícil a reprodução dos cenários apresentados em possíveis 
disciplinas de ABP.

A preferência por ferramentas de simulação genéricas indica que há espaço para expandir a utilização de simuladores específicos para RSSFs, que poderiam proporcionar uma visão mais completa de como as soluções propostas podem ser aplicadas. Neste ponto, técnicas de visualização do cenário simulado, assim como realidade virtual e aumentada, podem contribuir para a assimilação de problema e desenvolvimento de soluções.

\subsection{Limitações}

Essa seção apresenta algumas limitações como ameaças à validade do estudo de mapeamento sistemático. Na tabela 4 descrevem-se os tipos de ameaças a validade de acordo com (Petersen et al. 2015).

Tabela 4. Tipos de ameaças à validade

\begin{tabular}{|l|l|}
\hline Tipo de Ameaça & Descrição \\
\hline Descritiva & O quanto observações são corretas e objetivas. \\
\hline Teórica & $\begin{array}{l}\text { Estudos podem não ser encontrados na fase de busca, pesquisadores podem ser } \\
\text { parciais desde a identificação de estudos até sua classificação. }\end{array}$ \\
\hline Generalizabilidade & O quanto pode-se generalizar dentro e fora de grupos do tópico de pesquisa. \\
\hline Interpretativa & $\begin{array}{l}\text { As conclusões alcançadas de acordo com os dados disponíveis, também sofre risco } \\
\text { de pesquisadores serem parciais. }\end{array}$ \\
\hline Repetibilidade & O detalhamento do protocolo utilizado para a realização do mapeamento. \\
\hline
\end{tabular}

O estudo foi realizado utilizando um protocolo detalhado e mantendo todas as informações relevantes salvas. Para a extração de informações foi utilizado um formulário, assim seguindo as recomendações para garantir a validade descritiva e de repetibilidade.

Quanto a generalizabilidade, o estudo não pode ser generalizado para outros contextos de simulação além dos de agricultura.

Uma ameaça do tipo teórica surgiu na criação da string de busca quando foi utilizado o termo "simulation", o que pode ter resultado na perda de trabalhos relevantes. Porém, estudos sistemáticos baseados nessas buscas estruturadas apresentam tais características.

Outra ameaça à validade que se mostra inevitável é do tipo interpretativa. Como forma de mitigar os problemas causados pela parcialidade dos pesquisadores, foi feita uma validação das análises entre os autores do mapeamento.

\section{Conclusão}

A utilização de RSSFs na agricultura tem se mostrado essencial para atender as demandas da área, porém a utilização de dispositivos físicos para a realização de testes se torna inviável pelo alto custo e impraticabilidade em alguns cenários educativos.

A literatura atual carece de estudos sobre o uso de simulações que caracterizem bem os problemas nos domínios de RSSFs e agricultura. Ou seja, no geral, não são estudos prontos para utilização em disciplinas de ABP. Este artigo apresenta o resultado de um estudo de mapeamento sistemático sobre a área em uma análise que serve de motivação para que futuros trabalhos busquem mitigar esse lacuna da literatura da área. 
Os resultados encontrados levam a conclusão de que, apesar dos pontos levantados anteriormente, simulações não dedicam atenção suficiente para a problematização dos domínios nas pesquisas conduzidas. Os 35 trabalhos discutidos têm caráter mais científico, são desenhados com o intuito de testar as soluções propostas em simulação, e negligenciam demonstrações conceituais de um ambiente completo de sensoriamento. Portanto, há espaço para estudos direcionados para demonstrações conceituais na área, o que é essencial para utilização em disciplinas construídas nos preceitos de $\mathrm{ABP}$.

Por fim, sabe-se da necessidade de inovação na pesquisa de RSSFs aplicada para agricultura. No entanto, os desafios se tornam ainda mais aparentes para a aplicação dessas simulações em um ambiente acadêmico. Portanto, o uso de ABP em conjunto com simulações oferece uma oportunidade para enriquecer a pesquisa na área.

\section{Agradecimentos}

Este estudo foi parcialmente financiado pela Pró-Reitoria de Pesquisa (PROPESQ) e AGP, e pela FAPERGS por meio do projeto ARD N. 19 / 2551-0001268-3.

\section{Referências}

[Agrawal et al. 2019] Agrawal, H., Dhall, R., Iyer, K. S. S., and Chetlapalli, V. (2019). An improved energy efficient system for iot enabled precision agriculture.

[Banđur et al. 2019] Banđur, D., Jakšić, B., Banđur, M., and Jović, S. (2019). An analysis of energy efficiency in wireless sensor networks (wsns) applied in smart agriculture. Computers and Electronics in Agriculture, 156:500-507.

[Bayrakdar 2020] Bayrakdar, M. E. (2020). Employing sensor network based opportunistic spectrum utilization for agricultural monitoring. Sustainable Computing: Informatics and Systems, 27:100404.

[Bhanu et al. 2020] Bhanu, B. B., Husain, M. A., and Mirza, M. A. (2020). A high throughput offering iot system for agriculture applications.

[Castellanos et al. 2020] Castellanos, G., Deruyck, M., Martens, L., and Joseph, W. (2020). System assessment of wusn using nb-iot uav-aided networks in potato crops. IEEE Access, 8:56823-56836.

[Chen et al. 2015] Chen, Y., Chanet, J.-P., Hou, K.-M., Shi, H., and Sousa, G. (2015). A scalable context-aware objective function (scaof) of routing protocol for agricultural low-power and lossy networks (rpal). Sensors, 15:34.

[de Souza e Luis Dourado 2015] de Souza e Luis Dourado, S. C. (2015). Aprendizagem baseada em problemas (abp): Um mÉtodo de aprendizagem inovador para o ensino educativo. HOLOS, 5(0):182-200.

[Dhall and Agrawal 2018] Dhall, R. and Agrawal, H. (2018). An improved energy efficient duty cycling algorithm for iot based precision agriculture.

[Faid et al. 2020] Faid, A., Sadik, M., and Sabir, E. (2020). Iot-based low cost architecture for smart farming. In 2020 International Wireless Communications and Mobile Computing (IWCMC), pages 1296-1302. IEEE.

[Ibrahim et al. 2018] Ibrahim, H., Mostafa, N., Halawa, H., Elsalamouny, M., Daoud, R., Amer, H., Shaarawi, A., Khattab, A., and Elsayed, H. (2018). A high availability networked control system architecture for precision agriculture. 2018 International Conference on Computer and Applications (ICCA), pages 457-460. 
[Iqbal and Butt 2020] Iqbal, R. and Butt, T. (2020). Safe farming as a service of blockchain-based supply chain management for improved transparency.

[Jain 2020] Jain, J. K. (2020). A coherent approach for dynamic cluster-based routing and coverage hole detection and recovery in bi-layered wsn-iot. Wireless Personal Communications, 114.

[Jiang et al. 2018] Jiang, X., Yi, W., Chen, Y., and He, H. (2018). Energy Efficient Smart Irrigation System Based on 6LoWPAN: 4th International Conference, ICCCS 2018, Haikou, China, June 8-10, 2018, Revised Selected Papers, Part V, pages 308-319.

[Khan and Kumar 2019] Khan, F. and Kumar, D. (2019). Ambient crop field monitoring for improving context based agricultural by mobile sink in wsn. Journal of Ambient Intelligence and Humanized Computing.

[Khan et al. 2016] Khan, S., Pathan, A.-S. K., and Alrajeh, N. A. (2016). Wireless Sensor Networks: Current Status and Future Trends. CRC Press, Inc., Boca Raton, FL, USA, 1st edition.

[Khelifi 2020] Khelifi, F. (2020). Monitoring System Based in Wireless Sensor Network for Precision Agriculture, pages 461-472. Springer International Publishing, Cham.

[Lavanya et al. 2020] Lavanya, G., Rani, C., and GaneshKumar, P. (2020). An automated low cost iot based fertilizer intimation system for smart agriculture. Sustainable Computing: Informatics and Systems, 28:100300.

[Linsner et al. 2019] Linsner, S., Varma, R., and Reuter, C. (2019). Vulnerability assessment in the smart farming infrastructure through cyberattacks. In Meyer-Aurich, A., Gandorfer, M., Barta, N., Gronauer, A., Kantelhardt, J., and Floto, H., editors, 39. GIL-Jahrestagung, Digitalisierung für landwirtschaftliche Betriebe in kleinstrukturierten Regionen - ein Widerspruch in sich?, pages 119-124, Bonn. Gesellschaft für Informatik e.V.

[Mahalakshmi et al. 2020] Mahalakshmi, J., Kuppusamy, K., Kaleeswari, C., and Maheswari, P. (2020). Iot sensor-based smart agricultural system. In Subramanian, B., Chen, S.-S., and Reddy, K. R., editors, Emerging Technologies for Agriculture and Environment, pages 39-52, Singapore. Springer Singapore.

[Mukherjee et al. 2020] Mukherjee, A., Misra, S., Sukrutha, A., and Raghuwanshi, N. S. (2020). Distributed aerial processing for iot-based edge uav swarms in smart farming. Computer Networks, 167:107038.

[Musaazi et al. 2014] Musaazi, K., Bulega, T., and Lubega, S. (2014). Energy efficient data caching in wireless sensor networks: A case of precision agriculture. pages 154163.

[Nandhini et al. 2017] Nandhini, A., Rajendran, H., Sankararajan, R., and Indumathi, K. (2017). Web enabled plant disease detection system for agricultural applications using wmsn. Wireless Personal Communications.

[Nicolae et al. 2019] Nicolae, M., Popescu, D., Merezeanu, D., and Ichim, L. (2019). Large scale wireless sensor networks based on fixed nodes and mobile robots in precision agriculture. In Aspragathos, N. A., Koustoumpardis, P. N., and Moulianitis, V. C., editors, Advances in Service and Industrial Robotics, pages 236-244, Cham. Springer International Publishing.

[Ojha et al. 2017] Ojha, T., Misra, S., and Raghuwanshi, N. S. (2017). Sensing-cloud: Leveraging the benefits for agricultural applications. Computers and electronics in agriculture, 135:96-107.

[Petersen et al. 2008] Petersen, K., Feldt, R., Mujtaba, S., and Mattsson, M. (2008). Systematic mapping studies in software engineering. Proceedings of the 12th International Conference on Evaluation and Assessment in Software Engineering, 17. 
[Petersen et al. 2015] Petersen, K., Vakkalanka, S., and Kuzniarz, L. (2015). Guidelines for conducting systematic mapping studies in software engineering: An update. Information and Software Technology, 64:1 - 18.

[Shamshiri et al. 2020] Shamshiri, R. R., Bojic, I., van Henten, E., Balasundram, S. K., Dworak, V., Sultan, M., and Weltzien, C. (2020). Model-based evaluation of greenhouse microclimate using iot-sensor data fusion for energy efficient crop production. Journal of Cleaner Production, 263:121303.

[Shinghal et al. 2017] Shinghal, D., Srivastava, N., et al. (2017). Wireless sensor networks in agriculture: for potato farming. Neelam, Wireless Sensor Networks in Agriculture: For Potato Farming (September 22, 2017).

[Shipu et al. 2018] Shipu, X., Yunsheng, W., Yong, L., Weixiong, R., Mingzhou, M., Jingyin, Z., and Chenxi, Z. (2018). Research on wsn routing algorithm for vegetable greenhouse. pages $37-42$.

[Stewart et al. 2017] Stewart, J., Stewart, R., and Kennedy, S. (2017). Dynamic iot management system using k-means machine learning for precision agriculture applications. In Proceedings of the Second International Conference on Internet of Things, Data and Cloud Computing, ICC '17, New York, NY, USA. Association for Computing Machinery.

[Tanaka et al. 2018] Tanaka, K., Nishigaki, M., Sode, M., and Mizuno, T. (2018). Low delay data gathering method for rice cultivation management system: Iot specialized outdoor communication procedure. pages 139-143.

[Trotta et al. 2020] Trotta, A., Di Felice, M., Perilli, L., Scarselli, E. F., and Cinotti, T. S. (2020). Bee-drones: Ultra low-power monitoring systems based on unmanned aerial vehicles and wake-up radio ground sensors. Computer Networks, 180:107425.

[Uddin et al. 2019] Uddin, M. A., Ayaz, M., Aggoune, E. M., Mansour, A., and Jeune, D. L. (2019). Affordable broad agile farming system for rural and remote area. IEEE Access, 7:127098-127116.

[Uddin et al. 2017] Uddin, M. A., Mansour, A., Jeune, D. L., and Aggoune, E. H. M. (2017). Agriculture internet of things: Ag-iot. In 2017 27th International Telecommunication Networks and Applications Conference (ITNAC), pages 1-6.

[Uddin et al. 2018] Uddin, M. A., Mansour, A., Jeune, D. L., Ayaz, M., and Aggoune, E.-H. M. (2018). Uav-assisted dynamic clustering of wireless sensor networks for crop health monitoring. Sensors (Basel, Switzerland), 18(2).

[Wang et al. 2019] Wang, K. I.-K., Wu, S., Ivoghlian, A., Salcic, Z., Austin, A., and Zhou, X. (2019). Lws: A lorawan wireless underground sensor network simulator for agriculture applications. In 2019 IEEE (SmartWorld/SCALCOM/UIC/ATC/CBDCom/I$O P / S C I)$, pages $475-482$.

[Wu et al. 2020a] Wu, H., Li, Q., Zhu, H., Han, X., Li, Y., and Yang, B. (2020a). Directional sensor placement in vegetable greenhouse for maximizing target coverage without occlusion. Wireless Networks, 26.

[Wu et al. 2020b] Wu, S., Austin, A. C., Ivoghlian, A., Bisht, A., Kevin, I., and Wang, K. (2020b). Long range wide area network for agricultural wireless underground sensor networks. Journal of Ambient Intelligence and Humanized Computing, pages 1-17.

[Yassine et al. 2019] Yassine, S., Fatima, L., et al. (2019). Dynamic cluster head selection method for wireless sensor network for agricultural application of internet of things based fuzzy c-means clustering algorithm. In 2019 7th Mediterranean Congress of Telecommunications (CMT), pages 1-9. IEEE.

[Zhang et al. 2019] Zhang, M., Xiong, S., and Wang, L. (2019). Sensor-cloud based precision sprinkler irrigation management system. 